\title{
Introduction
}

\section{Cavernous malformations}

\author{
Steven L. Giannotta, M.D., ${ }^{1}$ and Daniel L. Barrow, M.D. ${ }^{2}$
}

${ }^{1}$ Department of Neurological Surgery, Keck School of Medicine, University of Southern California, Los Angeles, California; and ${ }^{2}$ Department of Neurological Surgery, Emory University Hospital, Atlanta, Georgia

Cavernous malformations are relatively rare vascular anomalies that occur in approximately $0.1 \%-4 \%$ of the population and account for $8 \%-15 \%$ of all vascular malformations of the CNS. These hamartomatous hemorrhagic lesions consist of cavernous spaces lined by epithelial cells and collagen. Our knowledge of the etiology, natural history, and indications for therapy were relatively primitive prior to the development of MR imaging. Unless associated with a recent hemorrhage or calcification, these lesions are difficult to diagnose on CT, and the minute feeding and draining vessels without arteriovenous shunting within the lesion make them angiographically invisible. Prior to the advent of MR imaging, even the nomenclature for cavernous malformations was confusing, including such vague terms as angiographically occult vascular malformations, cryptic malformations, cavernous hemangiomas, and cavernous angiomas. With the routine use of MR imaging, and the characteristic, if not nearly pathognomonic appearance, these lesions were readily identified in asymptomatic and minimally symptomatic patients. Furthermore, patients in whom doctors made misdiagnoses, such as demyelinating disease or neoplastic disorders, were able to be appropriately diagnosed with cavernous malformations. In the relatively short period of time since the advent and routine use of MR imaging, our knowledge of cavernous malformations has virtually exploded. The ability to accurately image cavernous malformations has led to a better understanding of the natural history of these lesions, the clinical manifestations, additional imaging characteristics, the role of microsurgical resection, the role of radiosurgical treatment, and even the genetics of cavernous malformations.

With such a rapid expansion of our database on this topic, it is incumbent on our scientific journals to periodically reassess that knowledge base and present it to our colleagues in a peer-reviewed and well-organized manner. This monograph attempts to accomplish that goal. We were fortunate to receive outstanding manuscripts from many of the world's authorities and experts in the biology, genetics, natural history, imaging, and therapeutic management of these interesting and complex lesions. Manuscripts by the Zhu, Couldwell, and Burkhardt groups update us on laboratory investigations into the pathogenetics of cerebral cavernous malformations with the Awad group proposing a novel theory relating pathogenesis to vascular permeability. Articles on the newest techniques for imaging and natural history observations lead into a virtual Who's Who of technical expertise discussing the surgical and radiosurgical management of eloquently positioned lesions. (DOI: 10.3171/2010.9. FOCUS.Intro) 